\title{
Enhanced Aluminum reflecting and solar-blind filter coatings for the far-ultraviolet
}

\author{
Javier Del Hoyo* and Manuel Quijada \\ NASA Goddard Space Flight Center, 8800 Greenbelt Road, Greenbelt, Maryland 20771, USA \\ *javier.delhoyo@nasa.gov
}

\begin{abstract}
The advancement of far-ultraviolet (FUV) coatings is essential to meet the specified throughput requirements of the Large UV/Optical/IR (LUVOIR) Surveyor Observatory which will cover wavelengths down to the $100 \mathrm{~nm}$ range. The biggest constraint in the optical thin film coating design is attenuation in the Lyman-Alpha Ultraviolet range of 100-130 $\mathrm{nm}$ in which conventionally deposited thin film materials used in this spectral region (e.g. aluminum [Al] protected with Magnesium fluoride $\left[\mathrm{MgF}_{2}\right]$ ) often have high absorption and scatter properties degrading the throughput in an optical system. We investigate the use of optimally deposited aluminum and aluminum tri-fluoride $\left(\mathrm{AlF}_{3}\right)$ materials for reflecting and solar blind band-pass filter coatings for use in the FUV. Optical characterization of the deposited designs has been performed using UV spectrometry. The optical thin film design and optimal deposition conditions to produce superior reflectance and transmittance using $\mathrm{Al}$ and $\mathrm{AlF}_{3}$ are presented.
\end{abstract}

Keywords: aluminum tri-fluoride, $\mathrm{AlF}_{3}$, aluminum, $\mathrm{Al}$, Fabry-Perot type filter, ultraviolet, FUV, dielectric metal filter, metal fluoride, band-pass filter, broadband.

\section{INTRODUCTION}

The Large Ultra-Violet/Optical/Infrared (LUVOIR) Surveyor is a large mission concept study announced by the NASA Astrophysics Division in 2016 that will allow for a plethora of scientific observations. ${ }^{1}$ In order to achieve these scientific objectives, the design will consist of a core telescope design that will be directing photons into instruments covering each respective spectral channel. Dissimilar to prior NASA flagship missions such as the Hubble Space Telescope (HST), the LUVOIR surveyor UV channel aims to capture the entirety of the Lyman-ultraviolet (LUV) spectral band (i.e. $91.2 \mathrm{~nm}-121.6 \mathrm{~nm}$ ) with a stretch goal of covering the $\sim 90 \mathrm{~nm}-300 \mathrm{~nm}$ range. ${ }^{2}$ The LUV spectral band is a highly desirable band in the astrophysics community due to its large spectroscopic line density. ${ }^{3}$

To understand the importance of thin film coatings, we can analyze the number of spectral photons going through an optical system and detector by considering the spectral radiant flux give as: $:^{4,5}$

$$
\Phi_{\lambda}=L_{\lambda} \cdot A_{E P} \cdot \Omega
$$

where $\lambda$ is the wavelength under observation, $\Phi_{\lambda}$ is the spectral radiant flux, $L_{\lambda}$ is the spectral radiance of the object or source at a chosen wavelength, $A_{E P}$ is the area of the entrance pupil, and $\Omega$ is the solid angle defined by the diameter and focal length of the imaging mirror. A baseline imaging spectrograph design is composed of a core Cassegrain telescope with a slit at the intermediate image/focal plane followed by a concave grating serving as both the dispersive and imaging element. ${ }^{6}$ The spectral radiant flux in Joules for a spatially resolved spectroscopic observation will then be given as:

$$
\Phi_{\lambda}=\tau_{\lambda} \cdot L_{\lambda} \cdot A_{E P} \cdot \Omega \cdot R_{\lambda, \text { optics }} \cdot \epsilon_{\lambda, \text { grat. }} \cdot Q_{\lambda, \text { det. }} \cdot \Delta \lambda \cdot \Delta t
$$

where $\tau_{\lambda}$ is the spectral transmission through the media of light propagation, $R_{\lambda, \text { optics }}$ is the total reflectance of the optics, $\epsilon_{\lambda, \text { grat. }}$ is the grating efficiency, $Q_{\lambda \text {,det. }}$ is the detector efficiency, $\Delta \lambda$ is the spectral bandwidth across the detector area, and $\Delta t$ is the integration time. (Note: There are sources of uncertainty such as polarization and scattered light not considered in this equation). 
It is exceptionally critical to increase the spectral radiant flux in the ultraviolet range since the smaller wavelengths are more subject to scatter and absorption traveling from an interstellar medium and through an optical system. Common techniques used to increase this value include designing the spectroscopic system so most of the optical elements are reflective, limiting the number of optical elements, and increasing the integration time. These methods, however, limit instrument design freedom and increasing integration time often leads to unwanted noise and compromises observational data. Therefore, it is imperative that high performance coatings be developed as the coatings can improve the total reflectance of the optics, the grating efficiency, as well as the detector efficiency.

In this paper, we present $\mathrm{Al}$ and $\mathrm{AlF}_{3}$ reflector and metal-dielectric Fabry-Perot type filter FUV coating design data which can be used in future LUVOIR instrument design. Technical background and our approach to choosing the materials for the broadband reflector and UV filter design is shown in Section 2. The thin film deposition method and measurement method is described in Section 3. The results and concluding remarks are respectively given in Sections 4 and 5 .

\section{BROADBAND COATINGS AND UV BAND-PASS FILTER DESIGN}

In order to meet the ultraviolet to infrared specifications, the core telescope will require a broadband coating covering the UV to infrared spectral ranges while the secondary instruments will contain coatings optimizing each respective channel while attenuating others. There are a variety of materials for thin film reflector and filter coating design in the visible and infrared spectral ranges, but the amount of materials that will limit photon loss in the deep ultraviolet below $\sim 120 \mathrm{~nm}$ is extremely limited. ${ }^{7}$ Therefore, the band-pass filters in this study will be designed for the UV.

\subsection{Reflecting broadband coatings}

Pure $\mathrm{Al}$ is the most prominent material to be used as a broadband reflector as it performs well from the UV to IR spectral ranges. ${ }^{8}$ However, it becomes oxidized following thin film deposition causing its FUV properties to severely degrade, thus it requires a thin overcoat of a transmissive protection layer to serve as a protection barrier as well as enhance its UV performance due to interference effects. ${ }^{9}, 10$ Thin metal fluoride overcoats (i.e. quarter wave) of $\mathrm{MgF}_{2}$ or lithium fluoride $(\mathrm{LiF})$ on aluminum are often used for $\mathrm{UV}$ applications since these materials have a high bandgap energy and a low cut off / absorption wavelength. ${ }^{11}$

The optical properties of these metal fluorides have recently been advanced as highly dense and reflective protected aluminum coatings with an intrinsic cut-off wavelength closer to the theoretical limit were developed using physical vapor deposition (PVD) and substrate heating. ${ }^{12}$ However, the $\mathrm{MgF}_{2}$ cut-off wavelength is near $115 \mathrm{~nm}$ while $\mathrm{LiF}$ is extremely hygroscopic leading to FUV optical property degradation over time. ${ }^{12,13}$ Therefore, it is necessary to find a more robust overcoat material with a smaller cut-off wavelength in order to meet LUVOIR coating requirements. $\mathrm{AlF}_{3}$ and $\mathrm{MgF}_{2}$ have been shown to have similar bandgap energy, ${ }^{14}$ so it is a viable replacement as a protective overcoat on aluminum.

\subsection{Fabry-Perot type band-pass filter design}

The use of a metal-dielectric Fabry-Perot type band-pass filter composed of a thin layer of aluminum and a metalfluoride could be used to suppress any unwanted photons from the visible spectrum and separate overlapping orders by the grating in the spectroscopic system. The Fabry-Perot type filter is based on the Fabry-Perot etalon, however, instead of an air gap between the reflective surfaces, a dielectric is substituted as a spacer layer. ${ }^{7}$ The Fabry-Perot filter works on the basis of the waves of reflected light from the successive interfaces of the cavity adding up out of phase and destructively interfering, causing only a certain wavelength or spectral band to transmit. Usually, the number of cavities (i.e. metal, dielectric, metal) is increased in filter design to increase the steepness of the band-pass edge cut-offs to more optimally attenuate unwanted wavelengths, and an extra dielectric layer is added over the final metal layer to prevent oxidation.$^{7,15}$ The study of these filters in the UV has performed in prior publications, however, the filter performance has not been validated below $\sim 175 \mathrm{~nm} .{ }^{15-17}$

$\mathrm{Al}$ and $\mathrm{AlF}_{3}$ are excellent candidates for the design of a metal, dielectric, metal, dielectric filters for the reasons stated above. The thin layer of $\mathrm{Al}$ will be used to suppress visible light while the low $\mathrm{UV}$ absorption properties of $\mathrm{AlF}_{3}$ will be used to optimize an FUV band-pass. Figure 1 shows the proposed Fabry-Perot type band-pass filter design using a MgF2 
substrate with $\mathrm{Al}$ as the metal layer and $\mathrm{AlF}_{3}$ as the dielectric layer. The number of layers is kept at a minimum to prevent absorption losses in the FUV and $\mathrm{MgF}_{2}$ is chosen as the substrate since it is the least hygroscopic/ hydrophilic metal fluoride with proven space flight heritage. ${ }^{18}$

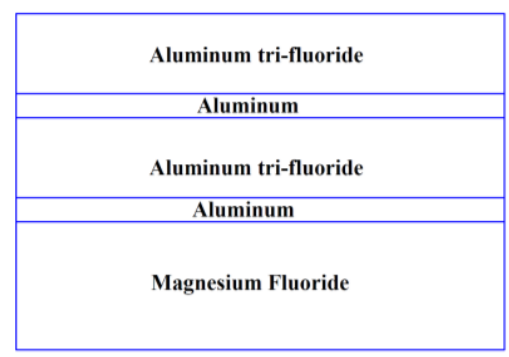

Figure 1. Metal dielectric Fabry-Perot type filter design using aluminum as the metal and aluminum tri-fluoride as the dielectric spacer layer deposited on a thin magnesium fluoride substrate.

\section{MATERIAL DEPOSITION AND OPTICAL CHARACTERIZATION}

\subsection{Physical vapor deposition}

High power thermal resistive evaporation in an ultra-high vacuum chamber was used to deposit the $\mathrm{Al}$ and $\mathrm{AlF}_{3}$ materials. High purity $\mathrm{Al}$ staples were wrapped around tungsten filaments while the $\mathrm{AlF}_{3}$ was placed into a molybdenum resistive bowl. The chamber was pumped to $<1 \times 10^{-7}$ torr before the process was started. Quartz crystal monitors were used to monitor the deposited material thickness and rate while high speed shutters over the depositing sources were used to control the final deposited thickness.

The three-step thermal resistive evaporation process is utilized for the deposition of the $\mathrm{Al}+\mathrm{AlF}_{3}$ reflecting films. ${ }^{12} \mathrm{~A} 70$ $\mathrm{nm}$ thin film of $\mathrm{Al}$ was deposited onto a $50 \mathrm{~mm} \times 50 \mathrm{~mm}$ quartz substrate at ambient temperature. The process was immediately followed by a thin $4 \mathrm{~nm}$ film of $\mathrm{AlF}_{3}$ as a protective layer before heating the substrate. Finally, the substrate was heated to $250^{\circ} \mathrm{C}$ before the final $\mathrm{AlF}_{3}$ layer was deposited with respective thicknesses of $20.5 \mathrm{~nm}$ and $20.1 \mathrm{~nm}$ for each experimental case. The deposition parameters are summarized in Table 1.

Table 1. Deposition parameters for deposited $\mathrm{Al}$ and $\mathrm{AlF}_{3}$ films

\begin{tabular}{llllll}
\hline $\begin{array}{l}\text { Material } \\
\text { Layer }\end{array}$ & $\begin{array}{l}\text { Deposition } \\
\text { Method }\end{array}$ & $\begin{array}{l}\text { Rate } \\
(\mathrm{nm} / \mathrm{sec})\end{array}$ & $\begin{array}{l}\text { Final Layer } \\
\text { Thickness }(\mathrm{nm})\end{array}$ & $\begin{array}{l}\text { Deposited Material } \\
\text { Purity } \%\end{array}$ & $\begin{array}{l}\text { Substrate } \\
\text { Temperature }{ }^{\circ} \mathrm{C}\end{array}$ \\
\hline $\mathrm{Al}$ & $\begin{array}{l}\text { Resistive } \\
\text { Evaporation }\end{array}$ & 10 & 70 & 99.999 & 27 \\
$\mathrm{AlF}_{3}$ & $\begin{array}{l}\text { Resistive } \\
\text { Evaporation }\end{array}$ & 2 & 4 & 99.500 & 27 \\
$\mathrm{AlF}_{3}{ }^{*}$ & $\begin{array}{l}\text { Resistive } \\
\text { Evaporation }\end{array}$ & 3 & $20.5,20.1$ & 99.500 & 250 \\
\hline
\end{tabular}

$\mathrm{AlF}_{3} *$ denotes a different $\mathrm{AlF}_{3}$ thickness parameter used for each respective deposition process.

To optically characterize the $\mathrm{AlF}_{3}$, a $24.5 \mathrm{~nm}$ layer of $\mathrm{AlF}_{3}$ was deposited on a $\mathrm{MgF}_{2}$ window which was manufactured with a cut along the optical axis to minimize birefringence. ${ }^{19}$ The substrate was heated to $250^{\circ} \mathrm{C}$ before the $\mathrm{AlF}_{3}$ layer was applied. The layer was deposited using same deposition parameters outlined in the final layer of $\mathrm{AlF}_{3}$ on Table 1.

\subsection{UV and visible optical characterization of deposited coatings}

The optical reflectance and transmittance measurements in the FUV spectral range were performed using a McPherson Vacuum Ultraviolet (VUV) AS 10X reflectometer. The instrument uses a hollow cathode gas purged light source to create a plasma covering the spectral lines of each respective gas fed into the source (e.g. hydrogen, neon, and helium). 
Radiation from the ionized gas then enters an entrance slit into a wavelength-tuning grating which relays the dispersed radiation into an exit slit, order sorting filters, and system aperture stop before reaching the element under test. Light can be reflected or transmitted from the element under test into photomultiplier tube (PMT) where the photons are absorbed and converted into electrons. The reflectometer used for the measurements is shown in Figure 2.

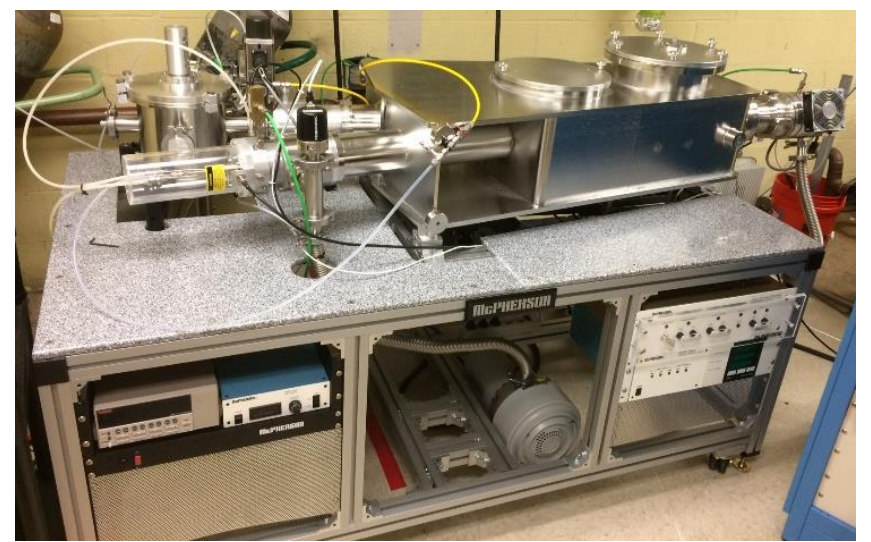

Figure 2. McPherson VUV reflectometer used for extreme ultraviolet and far ultraviolet (i.e. $58.4 \mathrm{~nm}$ to $200 \mathrm{~nm}$ ) reflectance and transmittance measurements.

An absolute measurement is performed by first taking a reference measurement without the element under test in the optical path of the radiation. The test element is then inserted into the optical path under transmission or reflection and a second measurement is obtained. For a transmittance measurement, the element under test is normal to the light source and PMT waveguide facet. A reflectance measurement consists of the element under test positioned at an angle of incidence of $10^{\circ}$ while the PMT waveguide facet is rotated and angled at $20^{\circ}$ in the optical path of the reflected light. The ratio of the radiation with the element under in the optical path over the radiation without the element under test in the optical path is then given as the respective transmittance or reflectance.

This $\mathrm{AlF}_{3}$ film was also optically characterized at larger UV to visible wavelengths with a Perkin Elmer Lambda 950 spectrometer. This spectrometer contains various channels capable of measuring the $190 \mathrm{~nm}$ to $2500 \mathrm{~nm}$ spectral range. This would allow of further wavelength characterization in the design of the proposed band-pass filter.

To derive the optical properties of the deposited $\mathrm{AlF}_{3}$ film, the $\mathrm{MgF}_{2}$ window to be coated was first measured in transmission and reflection. Using these values and the known thickness, the index of refraction, $n(\lambda)$, and extinction coefficient, $k(\lambda)$, of the window were extracted using a least squares fit to the theoretical Fresnel transmittance and reflectance equations with the FilmStar program. Through several iterations, the program adjusts the index of refraction and extinction coefficients as a function of wavelength until these values agree to the measured spectral reflectance and transmittance to $\pm 1 \%$. The reflectance and transmittance of the window were then re-measured after applying a quarter wave of $\mathrm{AlF}_{3}$ optimized at $121 \mathrm{~nm}$. The index of refraction and extinction coefficients of the $\mathrm{AlF}_{3}$ layer were then extracted using a similar least squares fit with the known optical properties of the window.

\section{ALUMINUM AND ALUMINUM TRI-FLUORIDE COATING RESULTS}

\subsection{Deposited $\mathrm{Al}$ and $\mathrm{AlF}_{3}$ broadband reflectors}

The viability of using $\mathrm{AlF}_{3}$ as an overcoat over $\mathrm{Al}$ to replace $\mathrm{MgF}_{2}$ and $\mathrm{LiF}$ as a broadband reflector was investigated in this study. The deposition parameters for depositing this coating design are outlined in Table 1 in Section 3.1. Unlike $\mathrm{MgF}_{2}$ and $\mathrm{LiF}$, when heating the $\mathrm{AlF}_{3}$ during thermal resistive evaporation, the substance sublimates. Thus reducing any pinholes that may arise during ejection of small un-melted source particles due to the gas accumulation in the confinements of the boat during the heating process. The FUV reflectance of two deposited $\mathrm{Al}_{+} \mathrm{AlF}_{3}$ designs is shown in Figure 3. 


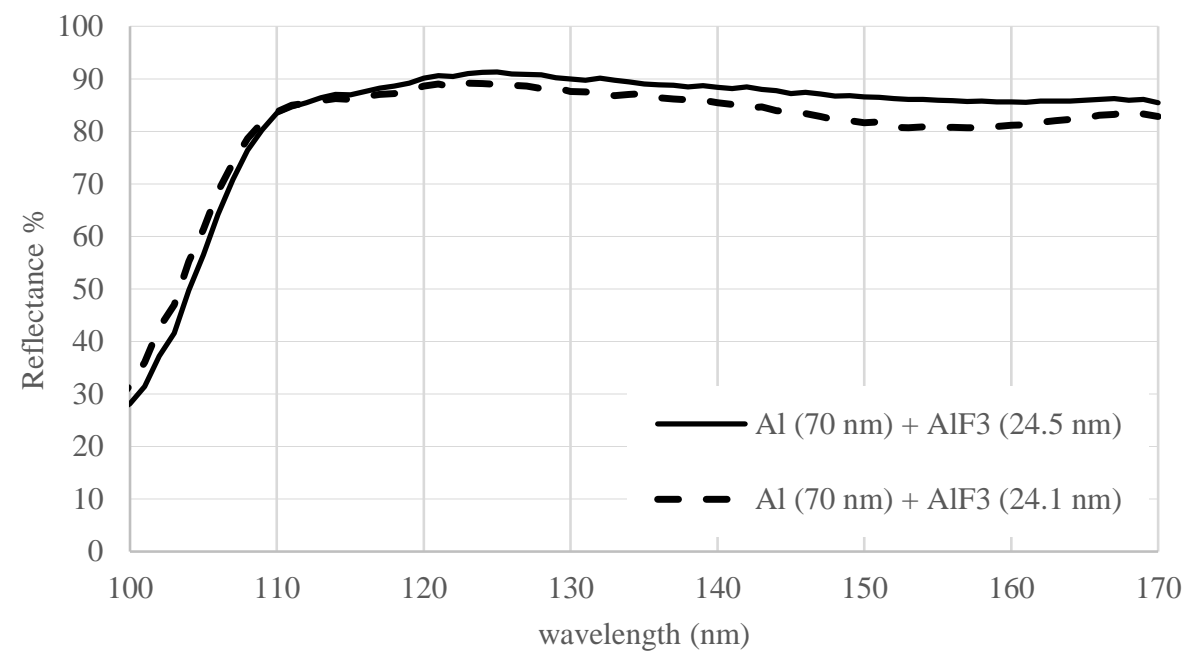

Figure 3. FUV Reflectance vs. Wavelength of deposited $\mathrm{Al}+\mathrm{AlF}_{3}$ samples.

It is evident that both designs produces optimal FUV reflectance results. Both designs maintained a reflectance of more than $80 \%$ from 109 to $170 \mathrm{~nm}$. The first sample with an $\mathrm{AlF}_{3}$ thickness of $24.5 \mathrm{~nm}$ peaked with a reflectance of $91.3 \%$ at $125 \mathrm{~nm}$ and maintained a high reflectance of more than $90 \%$ from $120 \mathrm{~nm}$ to $130 \mathrm{~nm}$. The second sample with the 24.1 $\mathrm{nm}$ thickness peaked at Lyman-Alpha $(121.6 \mathrm{~nm})$ with a reflectance of $89 \%$ and maintained slightly higher reflectance at smaller wavelengths starting at $110 \mathrm{~nm}$ while slightly underperforming the first sample at the larger wavelengths. The slight dip after the peak wavelength is attributed to slight interference effects.

\subsection{Derived $\mathrm{AlF}_{3}$ optical properties}

In order to design a suitable band-pass filter, the optical properties of the $\mathrm{AlF}_{3}$ layer were derived as described in section 3.2. The filter has to contain high UV transmission properties with strong rejection of visible light.
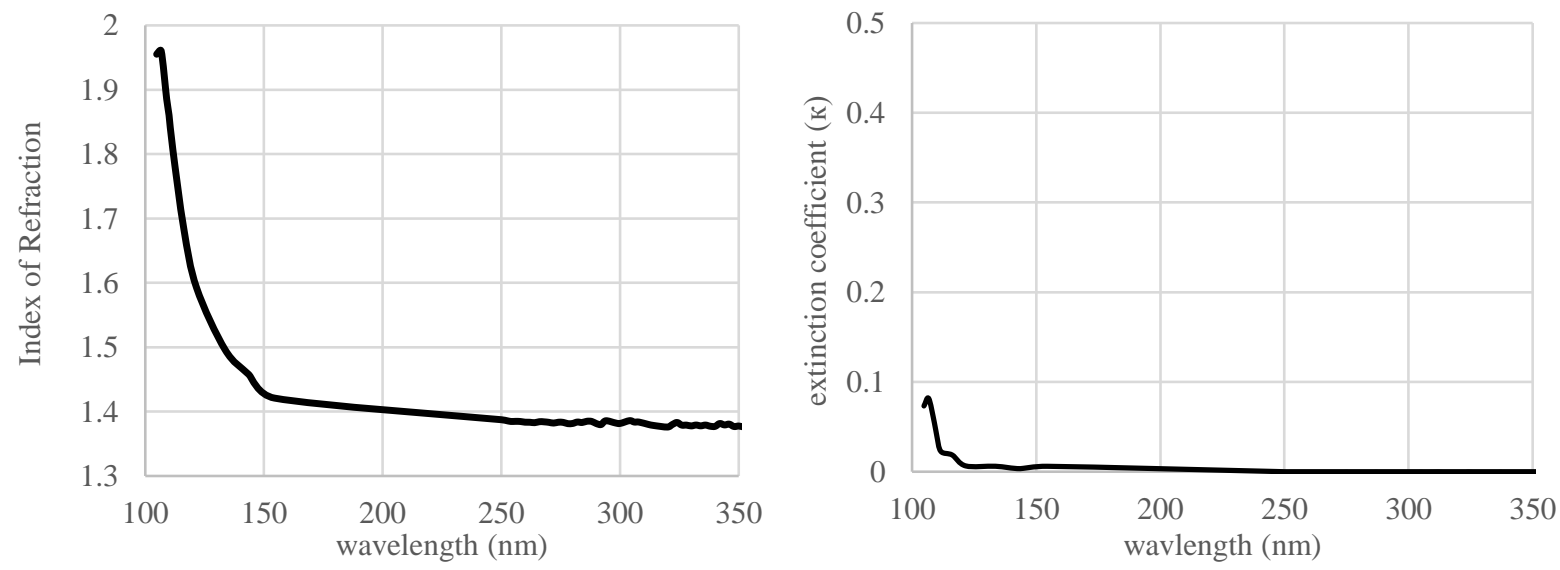

Figure 4. Index of refraction (left) and extinction coefficient (right) as a function of wavelength for $\mathrm{AlF}_{3}$ thin films.

As seen in Figure 4, the optical properties of $\mathrm{AlF}_{3}$ thin films show an increasing index of refraction and extinction coefficient as at the smaller FUV wavelengths. The index of refraction is between 1.4-1.38 from 200 to $350 \mathrm{~nm}$ while it exponentially increases at wavelengths smaller than $150 \mathrm{~nm}$ reaching an index of refraction of 1.96 at $107 \mathrm{~nm}$. The extinction coefficient stays near zero down to $116.2 \mathrm{~nm}$, then begins increasing at a higher rate nearly reaching .1 at $104.8 \mathrm{~nm}$. 


\subsection{Design of Fabry-Perot type FUV band-pass filter}

Using the optical properties in section 4.3, we are then able to design the proposed Fabry-Perot type FUV band-pass filter. The designed FUV band-pass filter using $\mathrm{MgF}_{2}$ as the substrate, $\mathrm{Al}$ as the metal layer, and $\mathrm{AlF}_{3}$ as the dielectric layer is displayed in Figure 5.

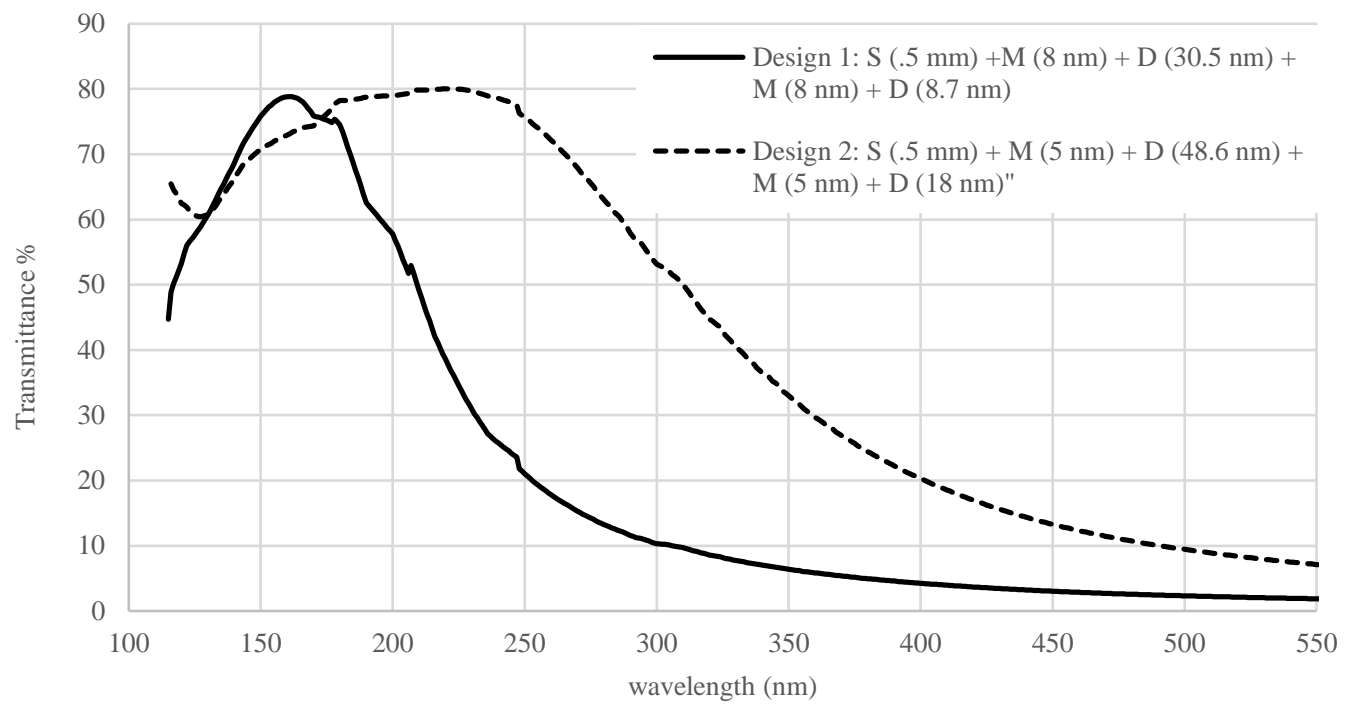

Figure 5. Four-layer Fabry-Perot FUV Filter designs consisting of an $\mathrm{MgF}_{2}$ substrate $(\mathrm{S}), \mathrm{Al}$ metal layers $(\mathrm{M})$, and an $\mathrm{AlF}_{3}$ dielectric layers (D).

Both designs peak near 80\%, however, Design 1 has a narrower band-pass and enhanced visibile attenuation than Design 2. Design 2 has an average transmittance near $80 \%$ from $175 \mathrm{~nm}$ to $250 \mathrm{~nm}$ while Design 1 maintains the same transmittance from $156 \mathrm{~nm}$ to $165 \mathrm{~nm}$. The design can be modified to meet band-pass and visible photon attenuation needs. By increasing the $\mathrm{Al}$ layer thickness, the visible spectrum is further suppressed and the full width half maximum (FWHM) band-pass of the filter is decreased. Design 1 and 2 have a FWHM of $105 \mathrm{~nm}$ and $218 \mathrm{~nm}$ respectively. Both designs are limited by the substrate material. $\mathrm{MgF}_{2}$ has a cut off wavelength near $115 \mathrm{~nm}$ causing $\mathrm{UV}$ absorption near that wavelength. Design 1 was modified so that the attenuation starts at wavelengths above $300 \mathrm{~nm}$ suppressing visible transmission to below 5\%.

\section{CONCLUDING REMARKS}

$\mathrm{AlF}_{3}$ is a low FUV absorbing coating that can be used in both FUV reflectors and band-pass coating design. FUV reflectors composed of $\mathrm{Al}+\mathrm{AlF}_{3}$ were deposited using thermal resistive evaporation and a reflectance of more than $~ 90 \%$ was obtained from $120 \mathrm{~nm}$ to $130 \mathrm{~nm}$. The $\mathrm{AlF}_{3}$ layer in the coating design can be deposited thinner to enhance the wavelengths below $110 \mathrm{~nm}$, however, the reflectance at the larger FUV wavelengths slightly decreases. $\mathrm{AlF}_{3}$ was shown to have an index of refraction near 1.4 from $150 \mathrm{~nm}$ to $350 \mathrm{~nm}$. The index of $\mathrm{AlF}_{3}$ at wavelengths under $150 \mathrm{~nm}$ exponentially increases to a value close to $2 . \mathrm{AlF}_{3}$ was shown to have very low absorbing properties down to $110 \mathrm{~nm}$. Using the material properties derived, we were able to design FUV solar blind band-pass filters that can meet FWHM band-pass and solar suppression requirements of future UV spectrograph needs.

\section{ACKNOWLEDGEMENTS}

We acknowledge the NASA Goddard Space Flight Center (GSFC) thin films lab for support of this work. We also would like to thank the NASA GSFC Internal Research and Development (IRAD) Astrophysics grant for funding this work.

\section{REFERENCES}


[1] Bolcar, M.R., Feinberg, L., France, K., Rauscher, B. J., Redding, D., Schiminovich, D., "Initial technology assessment for the Large-Aperture UV-Optical-Infrared (LUVOIR) mission concept study," Proc. SPIE 9904, Space Telescopes and Instrumentation 2016: Optical, Infrared, and Millimeter Wave, 99040J (July 29, 2016).

[2] Bolcar, M.R. et al., "Technology development for the Advanced Technology Large Aperture Space Telescope (ATLAST) as a candidate large UV-Optical-Infrared (LUVOIR) surveyor," Proc. SPIE 9602, UV/Optical/IR Space Telescopes and Instruments: Innovative Technologies and Concepts VII, 960209 (September 22, 2015).

[3] Tumlinson, J. et al., "Unique Astrophysics in the Lyman Ultraviolet,” ArXiv e-prints , Sept. 2012.

[4] Taubert, R. D. et al., "The Spectral Photon Flux of the Radiometric Calibration Source for the

NIRSpec Instrument of the James Webb Space Telescope,” Metrologia 46, S207-S212 (2009).

[5] F. Lei, W. Paustian, and E. Tegeler, "Determination of the spectral radiance of transfer standards in the spectral range $110 \mathrm{~nm}$ to $400 \mathrm{~nm}$ using BESSY as a primary source standard," Metrologia 32, 589-592 (1995).

[6] Fleming, B.T. et al., "SISTINE: a pathfinder for FUV imaging spectroscopy on future NASA astrophysics missions," Proc. SPIE 9905, Space Telescopes and Instrumentation 2016: Ultraviolet to Gamma Ray, 99050A (July 11, 2016).

[7] Macleod, H.A., [Thin-Film Optical Filters], Institute of Physics Publishing, $3^{\text {rd }}$ edition, Philadelphia, Chapters 3-7, 2001.

[8] Hass, G. "Filmed surfaces for reflecting optics," J. Opt. Soc. Am., 45, 945-52, 1955.

[9] Canfield, L. R., Hass, G. and Waylonis, J. E., "Further studies on MgF2-overcoated aluminium mirrors with highest reflectance in the vacuum ultraviolet," Appl. Opt., 5, 45-50, 1966.

[10] Cox, J. T., Hass, G. and Waylonis, J. E., "Further studies on LiF overcoated aluminium mirrors with highest reflectance in the vacuum ultraviolet," Appl. Opt., 7, 1535-9, 1968.

[11] Sommer, C., Kruger, P., and Pollmann, J., "Optical spectra of alkali-metal fluorides," Phys. Rev. B, 86 (15), 155212 (2012).

[12] M.A. Quijada, S. Rice, and E. Mentzell, "Enhanced MgF2 and LiF Over-coated Al Mirrors for FUV Space Astronomy," Proc. SPIE 8450 (2012).

[13] Wilbrandt, S., Stenzel, O., Nakamura, H., Wulff-Molder, D., Duparré, A., and Kaiser, N., "Protected and enhanced aluminum mirrors for the VUV," Appl. Opt. 53, A125-A130 (2014).

[14] Barrière, A.S. and Lachter, A., "Optical transitions in disordered thin films of the ionic compounds MgF2 and AlF3 as a function of their conditions of preparation," Appl. Opt. 16, 2865-2871 (1977).

[15] Mu, J., Lin, P.T., Zhang, L., Michel, J., Kimerling, L.C., Jaworski, F., and Agarwal, A., "Design and fabrication of a high transmissivity metaldielectric ultraviolet band-pass filter," Appl. Phys. Lett. 102, 213105 (2013).

[16] Bates, B. and Bradley, D.J., "Interference filters for the far ultraviolet,” Appl. Opt., 5, 971 (1966).

[17] Hennessy, J., Jewell, A. D., Hoenk, M. E., and Nikzad, S., "Metal-dielectric filters for solar-blind silicon ultraviolet detectors," Appl. Opt. 54, 3507-3512 (2015).

[18] Lallo, M.D., "Experience with the Hubble Space Telescope: 20 years of an archetype,” Opt. Eng., 51(1), 1-18 (2012).

[19] Sedlmeir, F., Hauer, M., Fürst, J.U., Leuchs, G., and Schwefel, H.G.L., "Experimental characterization of an uniaxial angle cut whispering gallery mode resonator," Opt. Express 21, 23942-23949 (2013). 
